\title{
Characterization of Small RNA Populations in Non-Transgenic and Aflatoxin-Reducing- Transformed Peanut
}

Imana L. Power ${ }^{1 *}$, Phat M. Dang ${ }^{1}$, Victor S. Sobolev ${ }^{1}$, Valerie A. Orner ${ }^{1}$, Joseph L. Powell ${ }^{1}$, Marshall C. Lamb ${ }^{1}$, Renee S. Arias ${ }^{1}$

${ }^{1}$ United States Department of Agriculture, Agricultural Research Service, National Peanut Research Laboratory (NPRL), 1011 Forrester Dr. S. E., Dawson, GA 39842, USA.

* Corresponding author. Tel.: +1 229995 7445; fax: +1 2299957416.

E-mail address: imanapower@yahoo.com (I. L. Power).

\begin{abstract}
Aflatoxin contamination is a major constraint in food production worldwide. In peanut (Arachis hypogaea L.), these toxic and carcinogenic aflatoxins are mainly produced by Aspergillus flavus Link and A. parasiticus Speare. The use of RNA interference (RNAi) is a promising method to reduce or prevent the accumulation of aflatoxin in peanut seed. In this study, we performed high-throughput sequencing of small RNA populations in a control line and in two transformed peanut lines that expressed an inverted repeat targeting five genes involved in the aflatoxinbiosynthesis pathway and that showed up to $100 \%$ less aflatoxin $B_{1}$ than the controls. The objective was to determine the putative involvement of the small RNA populations in aflatoxin reduction. In total, 41 known microRNA (miRNA) families and many novel miRNAs were identified. Among those, 89 known and 10 novel miRNAs were differentially expressed in the transformed lines. We furthermore found two small interfering RNAs derived from the inverted repeat, and 39 sRNAs that mapped without mismatches to the genome of $A$. flavus Power, Page 1, Plant Science
\end{abstract}


and were present only in the transformed lines. This information will increase our understanding of the effectiveness of RNAi and enable the possible improvement of the RNAi technology for the control of aflatoxins.

Keywords: Peanut, Groundnut, Aflatoxin, RNA interference, Small RNAs, miRNAs, siRNAs. Abbreviations: RNAi, RNA interference; miRNA, microRNA; siRNA, small interfering RNA; sRNA, small RNA.

\section{Introduction}

Aflatoxin contamination is a major constraint in the production of food worldwide. In the United States alone, aflatoxin contamination costs farmers and the peanut industry millions of dollars in losses each year [1]. Aside from causing high production costs, aflatoxins are a major health issue; they can cause acute hepatoxicity, immunosuppression [2], and stunting in children [3]. It is estimated that 4.5 billion people are chronically exposed to aflatoxins in the developing world [4]. Periodic outbreaks of aflatoxicosis occur due to ingestion of contaminated food and result in hundreds of human deaths in Asia and Africa $[5,6]$.

Furthermore, $58 \%$ of liver biopsies in cases of hepatocellular carcinoma have shown presence of aflatoxin $B_{1}\left(A f B_{1}\right)[7,8]$.

In peanut, aflatoxins are mainly produced by Aspergillus flavus Link and A. parasiticus Speare, but these pathogens can infect and produce aflatoxins in many other agriculturally important crops [9]. Aspergillus flavus produces $\mathrm{AfB}_{1}$ and $\mathrm{AfB}_{2}$, and $A$. parasiticus also produces aflatoxins $G_{1}$ and $G_{2}[10]$. Efforts to reduce aflatoxin accumulation in peanut have been focused on crop management practices and biocontrol, as well as on germplasm improvement aimed at 
resistance to drought and to infection by A. flavus [11-13]. Since the early 1960s, when aflatoxins were discovered, attempts to eliminate these mycotoxins in crops have failed, and no consistent effective method is available yet.

Induced gene silencing by RNA interference (RNAi) [14], the phenomenon where parasites in close contact with a plant producing gene-specific RNAi can receive a signal and undergo silencing of a target gene [15-17], has been demonstrated in many eukaryotes [18-20], and its potential use for crop improvement has been recently reviewed [21]. Though it is feasible to silence genes in fungal pathogens using plant-mediated RNAi, the control of aflatoxins is particularly challenging, and there are limited reports of its effective application $[22,23]$.

Several different enzymes are involved in the RNAi process: Dicer-like enzymes (DCLs) process double stranded RNA (dsRNA) into small RNAs (sRNAs); then one strand of the sRNA is loaded onto an Argonaute protein (AGO) to form the RNAi-Induced-Silencing Complex (RISC), which then mediates cleavage of RNA complementary to the sRNA, leading to silencing of that target gene. There are two main classes of sRNAs: microRNAs (miRNAs) and small interfering RNAs (siRNAs) [15-17]. MicroRNAs are derived from single-stranded RNA and are mostly processed by DCL1 into 19 to 24 nucleotides in Arabidopsis, whereas siRNAs are derived from dsRNA and cleaved into 20 to 24 nucleotides by DCL2, DCL3 or DCL4 [24-26]. In Arabidopsis, loading of sRNAs onto a specific AGO depends on the nucleotide at the 5 '-terminal end of the sRNA, for example, miRNAs with a uridine $(U)$ at their $5^{\prime}$ end are predominantly associated with AGO1, and miRNAs that start with an adenosine $(\mathrm{A})$ are predominantly associated with AGO2 and $\mathrm{AGO} 4$ [27]. 
Recently, Arias et al. [22] transformed peanut plants to generate dsRNA simultaneously targeting five genes in the aflatoxin biosynthesis pathway of $A$. flavus. In several of the transformed peanut lines obtained there was an 84 to $100 \%$ reduction in aflatoxin accumulation compared to the control. In order to gain insight into the putative involvement of SRNAs in the reduction of aflatoxin accumulation in peanut, we performed high-throughput sequencing of sRNAs from two transformed lines and compared the sRNA profiles to a control. We obtained 89 known miRNAs, two transgene-derived siRNAs, and we predicted 10 novel miRNAs that are putatively involved in the reduction of aflatoxin accumulation. This information enhances our understanding of RNAi of aflatoxin production, and will enable the possible improvement of RNAi technology for the control of aflatoxins.

\section{Materials and methods}

\subsection{Seeds}

Seed of RNAi-transformed and control peanut plants were used in this study. Transformed peanut lines (288-10 and 288-11) were obtained through Agrobacterium-mediated transformation, by integrating dsRNA targeting five genes involved in aflatoxin biosynthesis (RNAi-5x) as previously reported [22]. The five genes were AFL2G_07223 (aflS or aflJ), AFL2G_07224 (aflR), AFL2G_07228 (aflC/ pksA/ pksL1), AFL2G_07731 (pes1), and AFL2G_05027 (aflatoxin efflux pump, aflep); numbers correspond to accessions in A. flavus genome annotation (BROAD Institute, Cambridge MA), other names are in parentheses [28]. Peanut line 288-9 went through the process of transformation but did not carry the RNAi-5X insert or the selectable marker neomycin phosphotransferase II (NPTII), and was used as a control. The hull-scrape method [29] was used to determine maturity stages of pods, and first-generation 
immature and mature seed ( $T_{0}$ seed) harvested from transformed plants and control plants, were used for RNA extractions and challenging experiments.

\subsection{Detection and expression of the selectable marker NPTII and the RNAi-5x insert}

For the detection and expression analyses, total RNA was extracted from non-inoculated half cotyledons of immature and mature seeds, after 24 and $48 \mathrm{~h}$ incubation, using RNeasy Plant Mini Kit (Qiagen, CA) in the Qiacube robot. Complementary DNAs were synthesized using $1 \mu \mathrm{g}$ of RNA per sample, combining random hexamers and oligo-dT in Superscript III First Strand Synthesis Super Mix (Invitrogen, MA). Detection of the selectable marker NPTII was carried out using single-tube nested PCRs (STN-PCR), as described by Gomes et al. [22] with modifications by Arias et al. [23], using external primers PCAPD 5714F: 5'-AGGCTATTCGGCTATGACTG-3' and PCAPD 6446R: 5'-CGTCAAGAAGGCGATAGAAG-3', and internal primers PCAPD 5730F: 5'ACTGGGCACAACAGACAATC-3' and PCAPD 6249R: 5'-ATATTCGGCAAGCAGGCATC-3'. Briefly, the STN-PCR involves two PCR reactions, performed in one tube, in $20 \mu$ l total volume, containing 4 $\mu$ l of $5 x$ Phire reaction buffer (Carlsbad, CA), 1.25mM dNTPs, $0.4 \mu$ l Phire Hot start II DNA polymerase (Carlsbad, CA), $10 \mathrm{pmol}$ of each forward and reverse external primers, $1.0 \mu \mathrm{I}$ DNA template, and 13.4 $\mu$ l RNase-DNase free water. Before amplification, $100 \mathrm{pmol}$ of each forward and reverse internal primers were dried with bromophenol blue in the cap of a $0.5-\mathrm{ml} \mathrm{PCR} \mathrm{tube,}$ by incubating the tube at $37^{\circ} \mathrm{C}$. The amplification conditions consisted of 2 rounds of an initial denaturing step at $98^{\circ} \mathrm{C}$ for 30 , followed by 22 cycles of $98^{\circ} \mathrm{C}$ for $5 \mathrm{~s}, 65^{\circ} \mathrm{C}$ for $5 \mathrm{~s}$ and $72^{\circ} \mathrm{C}$ for $15 \mathrm{~s}$, and a final extension at $72^{\circ} \mathrm{C}$ for $60 \mathrm{~s}$. After this first round, the tubes were inverted to dissolve the internal primers that had been immobilized in the cap, and the reactions were placed back into the thermal cycler to continue with the next-cycle round, using the same 
conditions. The PCR products were separated on a $1 \%$ agarose, visualized by staining with ethidium bromide, and photographed over UV light using the Kodak Gel logic 200 Imaging System.

To detect the RNAi-5x insert, primers 35S-PDSFw: 5'-CCTAACAGAACTCGCCGTAA-3' and DirAll- Nco-Rv: 5'-ATGCCATGGGGTTATTGGGTGCAGAATGG-3' were used with genomic DNA, extracted from peanut leaves of putatively transformed plants. The PCR reaction, $20 \mu$ total volume, contained $4 \mu \mathrm{l}$ of $5 x$ Phire reaction buffer (Carlsbad, CA), 1.25mM dNTPs, $0.4 \mu$ I Phire Hot start II DNA polymerase (Carlsbad, CA), 10 pmol of each forward and reverse primer, $1.0 \mu \mathrm{l}$ DNA template, and $13.4 \mu \mathrm{l}$ RNase-DNase free water. The amplification conditions were an initial denaturing step at $98^{\circ} \mathrm{C}$ for $30 \mathrm{~s}$ followed by 40 cycles of $98^{\circ} \mathrm{C}$ for $5 \mathrm{~s}, 63^{\circ} \mathrm{C}$ for $5 \mathrm{~s}$ and $72^{\circ} \mathrm{C}$ for $15 \mathrm{~s}$, and a final extension at $72^{\circ} \mathrm{C}$ for $60 \mathrm{~s}$. Agrobacterium tumefacium $\mathrm{C} 58 \mathrm{C} 1$ containing plasmid p5xCAPD, that harbored the RNAi-5x-insert, was used as a positive control, at 2- and 10-fold dilutions, with or without plant tissue (to test for possible PCR inhibitors). The PCR products were separated on a $1 \%$ agarose, visualized by staining with ethidium bromide, and photographed over UV light using the Kodak Gel logic 200 Imaging System.

Expression of the RNAi-5x insert was analyzed by quantitative- reverse- transcriptase PCR (qRT-PCR) on ABI 7500 (Applied Biosystems, CA). For detection of expression of the selectable marker NPTII, primers RT_NPTII_1_6871F: 5'-CTCGCTCGATGCGATGTTTC-3' and RT_NPTII_1_7004R: 5'-GCAGGATCTCCTGTCATCTC-3' were used. Two sets of primers were used to detect expression of the RNAi-5x insert: RT_5x_1_105F: 5'-GGTGGCATTGGACCGTCTTG-3' and RT_5x_1_232R: 5'-CGCATCGAGGACAGGTTGTG-3'; and RT_5x_2_95F: 5'-

CCATGTTTCTGGTGGCATTG-3' and RT_5x_2_229R: 5'-ATCGAGGACAGGTTGTGTTG-3'. The 
peanut housekeeping gene actin was used as reference gene, using primers Actin- Fw: 5'CACATGCCATCCTTCGATTG-3' and Actin-Rv: 5'-CCAAGGCAACATATGCAAGCT-3' [31]. The qRTPCR reaction, $24 \mu \mathrm{l}$ total volume, contained $12 \mu \mathrm{l}$ of $\mathrm{RT}^{2}$ SYBR Green qPCR Mastermix (Qiagen, CA), $10 \mathrm{pmol}$ of each forward and reverse primer, and $5.0 \mu \mathrm{l}$ of a 1:6 dilution of cDNA template. The amplification conditions were $50^{\circ} \mathrm{C}$ for $2 \mathrm{~min}, 95^{\circ} \mathrm{C}$ for $10 \mathrm{~min}$, followed by $45 \mathrm{cycles}$ of $95^{\circ} \mathrm{C}$ for $15 \mathrm{~s}, 58^{\circ} \mathrm{C}$ for $60 \mathrm{~s}$, and $72^{\circ} \mathrm{C}$ for $60 \mathrm{~s}$. The numbers of cycles for $\mathrm{C}_{\mathrm{T}}=1$ were calculated by regression and the fold change was expressed as Log2 of the numbers of cycles, as previously reported [32].

\subsection{Seed inoculations and aflatoxin analysis}

First-generation immature and mature seeds harvested from transformed and control plants, were challenged with A. flavus isolate NRRL 3357 (provided by Dr. Bruce Horn), as described by Arias et al. [22]. Briefly, seeds were surface sterilized and imbibed in sterile deionized water, after which the seed coats and embryos were removed. The cotyledons were cut in half, placed with the cut side up on $1.5 \%$ water agar, and inoculated with $2 \mu$ of $A$. flavus spore suspension of $10 \times 10^{6}$ spores $\mathrm{ml}^{-1}$. Plates containing the inoculated and non-inoculated half cotyledons were incubated at $30^{\circ} \mathrm{C}$ for 24,48 and $72 \mathrm{~h}$.

Cleanup procedure and Ultra-Performance Liquid Chromatography (UPLC) of aflatoxins in experimental and control samples were performed as described by Sobolev and Dorner [33] with modifications as described by Arias et al. [23]. Briefly, seeds were extracted with methanol followed by a cleanup procedure on a mini column packed with basic aluminum oxide. Aflatoxins were separated in a UPLC instrument equipped with a matching UPLC Quaternary Solvent Manager, UPLC Sample Manager, UPLC Fluorescent Detector, and a C18 2.1 
$\mathrm{mm} \times 50 \mathrm{~mm}, 1.7 \mu \mathrm{m}$ column. Aflatoxin identity was determined by obtaining their UV and mass-spectral data and comparing them with published data [34]. Aflatoxin concentrations in samples were determined by reference to peak areas of commercial standards.

\subsection{High throughput sequencing, annotation, and differential expression of small RNAs}

Non-inoculated half cotyledons of immature and mature seeds from transformed lines 288-10 and 288-11, and control line 288-9, were sampled after 24 and $48 \mathrm{~h}$ of incubation. Total RNA was extracted from the half cotyledons using Trizol reagent (Invitrogen, MA) according to manufacturer's protocol. High-throughput sequencing was performed by LC Sciences (Houston, TX) on the Illumina GAllx platform, and 12 non-replicated libraries were obtained. Unmappable reads (reads containing adapter sequences and reads shorter than 15 nucleotides), as well as reads that mapped to mRNA, Rfam or Repbase were discarded. Mappable reads, ranging from 15-45 nucleotides in length were mapped to pre-miRNA and mature miRNAs in miRBase v21.0, using Bowtie 2.0, to identify known miRNAs. MicroRNAs with a minimum read count of 100 in at least one of the libraries were considered in the analyses.

To identify potentially novel miRNAs, the reads were aligned with the genomes of the diploid parents (Arachis duranensis and A. ipaënsis; www.peanutbase.org) of cultivated peanut. The following parameters were used: number of allowed errors in one bulge in stem $\leq 12$; number of basepair (bp) in stem region $\geq 16$; minimum free energy ( $\mathrm{dG}$ in $\mathrm{kCal} / \mathrm{mol}$ ) $\leq-15$, length of hairpin (up and down stem + terminal loop) $\geq 50$; length of terminal loop $\leq 350$; number of allowed errors in one bulge in mature region $\leq 8$; number of allowed biased errors in one bulge in mature region $\leq 4$ number of allowed biased bulges in mature region $<=2$; number of basepair (bp) in mature or mature* region: $\geq 12$; percentage of small RNA in stem region 
$(p m) \geq 80 \%$; number of allowed errors in mature region $\leq 7$. In order to identify siRNAs that possibly derived from the transgene, reads that did not map to miRNAs in miRBase or $A$. duranensis and $A$. ipaënsis were mapped to the RNAi-5x insert and the $A$. flavus genome. Potential target genes were predicted using the software program psRNATarget (http://plantgrn.noble.org/psRNATarget/) [35].

Normalized reads were used to determine the differential expression of the sRNAs. The fold-change between transformed and control peanut was calculated as: fold-change $=$ Log2 (transformed/ control). Small RNAs were considered upregulated if Log2 $\geq 2$ and downregulated if $\log 2 \leq 2$. Heat maps were constructed using the software program CIMminer (https://discover.nci.nih.gov/cimminer/home.do)

\section{Results}

\subsection{Detection and Expression of RNAi-5x insert and NPTII}

The selectable marker NPTII and the RNAi-5x insert were detected in both transformed lines, 288-10 and 288-11. Examples of PCR detection of NPTII and the RNAi-5x insert in transformed peanut lines are shown in Fig. $1 \mathrm{~A}$ and $1 \mathrm{~B}$, respectively. The 500 bp band, representing the presence of NPTII, was present in the positive control (plasmid DNA of Agrobacterium harboring plasmid p5xCAPD that contained the RNAi-5x-insert), and in the positive plant; no band was detected in the negative control (Fig. 1 A). The 931 bp band represented the presence of the RNAi-5x-insert, and was present in the positive controls (Agrobacterium harboring plasmid p5xCAPD that contained the RNAi-5x-insert, at different DNA concentrations, with or without plant tissue added), as well as in the positive plants. No band was detected in the negative control (Fig. $1 \mathrm{~B}$ ). Expression of the RNAi-5x insert was only detected in the 
immature seeds of transformed lines 288-10 and 288-11; the control was negative (Fig. 1 C).

High levels of expression of the RNAi-5x insert were observed by Real-Time PCR in line 288-10 and low levels in 288-11, after $24 \mathrm{~h}$ incubation; no expression of the RNAi-5x insert was detected at $48 \mathrm{~h}$ incubation in any of the lines (Fig. $1 \mathrm{~B}$ ).

\subsection{Aflatoxin analysis}

To test the effectiveness of RNAi-mediated potential control of aflatoxin accumulation, we directly applied conidia of $A$. flavus NRRL 3357 on cut half cotyledons from which the embryos and testa had been removed. In the first experiment only few seeds were available, so they were not separated by maturity stages. The level of $A f B_{1}$ and $A f B_{2}$ after $72 \mathrm{~h}$ incubation was 92\% and 95\% lower than the control, respectively, with highly significant differences, Tukey's test $P<0.009$ for $\mathrm{AfB}_{1}$ and $P<0.001$ for $\mathrm{AfB}_{2}$ (Fig. 2). When sufficient seeds were available, the seeds were separated into two maturity groups by yellow and brown mesocarp (immature and mature seed, respectively). In mature seeds of line 288-10, the levels of $A f B_{1}$ (Fig. $3 A$ ) and $A f B_{2}$

(Fig. 3 C) were $80 \%$ and $78 \%$ lower, respectively, compared to the control, however, these differences were not statistically significant (Fig. 3 A, Fig. 3 C). The immature seed, however, showed highly significant differences in the level of $\mathrm{AfB}_{1}$ of both transformed lines $288-10$ and 288-11 at 48 and $72 \mathrm{~h}$ incubation, compared to the control, Tukey's test $P<0.004$ and $P<$ 0.002, respectively (Fig. 3 B). The overall reduction in the level of $A^{\prime} B_{1}$ in immature seed at 48 and $72 \mathrm{~h}$ was $100 \%$ and $100 \%$ for line $288-10$, and $98 \%$ and $98 \%$ for line $288-11$, respectively

(Fig. 3 B). Highly significant differences were also found for both transformed lines, compared to the control for the level of $\mathrm{AfB}_{2}$ at 48 and $72 \mathrm{~h}$, Tukey's test $P<0.001$ and $P<0.011$, 
respectively (Fig. 3. D). The overall reduction in the level of $A f B_{2}$ in immature seed at 48 and 72 h was $100 \%$ and $98 \%$ for line $288-10$, and $97 \%$ and $99 \%$ for line $288-11$, respectively (Fig. 3 D).

\subsection{High throughput sequencing of small RNAs}

Twelve non-replicated small RNA (sRNA) libraries were generated from immature (I) and mature (M) seeds of control (288-9) and transformed (288-10 and 288-11) peanut lines, at two incubation periods ( 24 and $48 \mathrm{~h}$ ). After removal of adapters and low quality reads, and retaining reads of 15 to 45 nucleotides (nt) in length, the obtained mappable reads ranged from 6.9 million to 14.4 million reads for each of the libraries (Fig. 4). The abundance of sRNAs in mature seeds of control line 288-9 (288-9M) was higher than in immature seeds of this line (288-9I), at both incubation times, Fig. 4A-B. The opposite occurred for the libraries of the transformed lines, where the abundance of sRNAs in immature seeds of lines 288-10 and 28811 (288-10I and 288-11I) was much higher than in mature seeds of these lines (288-10M and 288-11M), respectively (Fig. 4C-F). For all 12 libraries, the majority of sRNAs were 21 or $24 \mathrm{nt}$ in length, and had uridine $(U)$ or adenine $(A)$ as $5^{\prime}$ terminal nucleotide, with percentages ranging from 32 to $45 \%$, while the percentage of sRNAs that had cytosine (C) or guanine (G) ranged from 12 to $21 \%$. This phenomenon was similar for transformed and control lines.

\subsection{Abundance and expression profiles of known peanut miRNAs}

To identify known miRNAs, the sRNA libraries were mapped to plant miRNAs in miRBase ( $v$ 21.0). There were 41 known miRNA families identified in the libraries of the transformed and control peanut lines. Several of the miRNA families identified contained multiple members, but the majority of the miRNA families, 19 , were represented by just one member (Table A. 1 ). The miR166 family was represented by the largest number of members (12), followed by miR156, 
with 10 members. Expression levels of members of the same miRNA family differed drastically, for example read counts of miR166 ranged from 1 or 2 reads to more than 167,000 reads in the different libraries (Table A. 1).

Thirteen of the 21 peanut miRNAs annotated in miRBase were detected in the transformed and control peanut libraries. The miRNAs not detected were miR394, miR3510, miR3512, miR3515, miR3517, miR3518, miR3519, and miR3520. Furthermore, miR162, miR166, miR168, miR169, miR171, miR172, miR390, miR894, miR1507, miR1511, and miR2118 are not reported in miRBase as peanut miRNAs, but have been reported by Chi et al. [36] and Zhao et al. [37], and these miRNAs were detected in our libraries as well (Table A. 1, A. 2). The miRNA families miR319, miR2592, miR2673, miR2916, miR5072, miR5083, miR5368, miR5538, miR5652, miR5724, miR6025, miR6108, miR6300, miR6478,8175, and miR9410 have not been reported in peanut before. Three miRNA families were present in very high abundance, with more than 10,000 read counts in at least one library; these were miR159, miR166, and miR482. Seventeen miRNA families were in medium high abundance, with more than 1000 read counts in at least one library: miR156, miR167, miR168, miR319, miR408, miR894, miR1507, miR1511, miR2118, miR2916, miR3508, miR3511, miR3514, miR3516, miR5368 and miR6300, miR8175. (Table A. 2).

To determine the miRNAs that may be associated with reduction in aflatoxin production, we compared the fold-change of miRNAs in the libraries of transformed and control lines. We found that 39 of the 41 miRNA families may be involved in suppression of aflatoxin production: the miRNAs belonging to the miRNA families miR156, miR159, miR160, miR162, miR166, miR167, miR169, miR171, miR172, miR319, miR390, miR398, miR408, miR482, 
miR894, miR1507, miR1511, miR2118, miR2592, miR2673, miR2916, miR3508, miR3509, miR3513, miR3514, miR3516, miR3521, miR5072, miR5083, miR5368, miR5538, miR5652, miR5724, miR6025, miR6108, miR6300, miR6478, miR8175, and miR9410, were differentially expressed (either upregulated or downregulated) (Table A. 3, Fig. 5). Some miRNAs were exclusively differentially expressed in a specific line, seed maturity, or incubation time (regardless of seed maturity): ahy-miR166a-3py, ahy-miR166a-3pz, ahy-miR166a-3px, and ahymiR166a-3p were downregulated only in immature seed of line 288-10, incubated 24h (28810I-24), and ahy-miR156b-5p was only upregulated in 288-10I-24. The miRNAs ahy-MIR156ep3x, ahy-MIR156c-p3x, ahy-miR6300xb, ahy-miR6300xa, ahy-miR6300z, and ahy-miR482b-3pz were downregulated in 288-10l-24 but upregulated at $48 \mathrm{~h}$ incubation in both lines, regardless of maturity. The miRNA ahy-MIR3509-p5x was only differentially expressed (upregulated) at 48h incubation in both $288-10$ and $288-11$, in both immature and mature seed. Some miRNAs were downregulated in immature seed, but upregulated in mature seed: ahy-miR894z, ahymiR894y, ahy-miR894x, ahy-miR5072x, ahy-MIR5724-p3x, ahy-MIR5652-p3x, ahy-MIR5652-p5x, ahy-MIR5652-p5y, and ahy-MIR6025c-p3y were downregulated in immature seed of line 28810 (288-10I), but upregulated in mature seed $48 \mathrm{~h}$ incubation of both transformed lines 288 -

10M-48 and 288-11M-48 (Fig. 5). The target genes of the differentially expressed miRNAs are related to plant development, morphology or flowering time, transcription factors, and plant metabolism (Table A. 2).

\subsection{Expression profiles of putatively novel miRNAs}

To identify novel miRNA candidates, the reads that did not map to miRNAs in miRBase were aligned to the diploid progenitors of cultivated peanut, $A$. duranensis and $A$. ipaënsis 
(www.peanutbase.org), since the genome of $A$. hypogaea is unavailable. In total 70 potential novel miRNA candidates were identified in the different libraries (data not shown). To identify novel miRNAs that possibly contribute to the reduction of aflatoxin production in the transformed lines, miRNAs with the $[\log 2] \geq 2$, and a minimum read count of 100 in at least one of the libraries, and that have no errors in the mature region were identified; 10 novel miRNAs fit these conditions (Table 1, Table A. 4). All novel miRNAs were upregulated after $24 \mathrm{~h}$ incubation but downregulated after $48 \mathrm{~h}$ incubation in both immature and mature seeds of line 288-10 (Fig. 6). In order to determine the functions of the predicted novel miRNAs, their targets were predicted using psRNATarget (http://plantgrn.noble.org/psRNATarget/) [35]; ahynovel-2 putatively targets drought-responsive family protein, and ahy-novel-4 putatively targets disease resistance proteins. Other targets are related to regulatory mechanisms, plant metabolism, and transcription factors (Table A. 5).

\subsection{Small RNAs homologous to the RNAi-5x insert and A. flavus}

Since the RNAi-5x insert was constructed from five $A$. flavus genes involved in aflatoxin biosynthesis, to identify siRNAs involved in reduction of aflatoxin production we mapped the reads that did not map to miRBase or the $A$. duranensis and $A$. ipaënsis genomes to the RNAi-5x insert and to the $A$. flavus genome. We found two siRNAs matching the RNAi-5x insert, mapping to gene fragments aflS/ aflJ and aflC/ pksA (Table 2). In addition, 34 sRNAs, 20- 24 nt in length, were perfect matches to the A. flavus genome, and only present in the transformed lines, however, the abundance of these sRNAs was low, 7 reads or less (Table 3). 


\section{Discussion}

This is the first report on small RNA sequencing of genetically transformed peanut plants for silencing five genes in the aflatoxin-biosynthesis pathway of $A$. flavus, obtained as described by Arias et al. [22]. Research on plant-induced RNAi silencing of single fungal genes has shown reduction of aflatoxin levels in the range of 60 and 97\% [17, 24, 38]. Masanga et al. [24] demonstrated a 14-fold reduction in aflatoxin accumulation in transformed maize, expressing a hairpin construct targeting afIR. In our study the cotyledons of transformed peanut challenged with $A$. flavus, exhibited between up to $100 \%$ lower levels of $\mathrm{AfB}_{1}$, and between 74 and $100 \%$ lower levels of $\mathrm{AfB}_{2}$ than the control. Line 288-10 showed consistently low levels of aflatoxins, whereas line 288-11 had overall lower levels of aflatoxins during the first $48 \mathrm{~h}$ incubation (Figures 2-3). Differences found between transformed lines 288-10 and 288-11 were not surprising, since plant biotechnology using RNAi usually showed wide variations between transformed events, both in silencing and phenotypes (reviewed by McGinnis [39]).

Our data indicate that the enzymes involved in RNAi were active. In Arabidopsis, 21-nt sRNAs are produced by DCL4, 24-nt sRNAs by DCL 2 or DCL 3, and DCL1 produces miRNAs that are 22 nucleotides in length $[28,40]$. In this study a large percentage of the sRNAs had read lengths of 20 to 24 nucleotides, which is typical for DICER-processed sRNA products [25-27].

The size distribution of the sRNAs in peanut is also consistent with previous reports [36, 37]. Furthermore, the majority of sRNAs had a uracil or adenine as $5^{\prime}$ terminal nucleotide, which suggest preferential loading onto AGO1, and AGO2 and AGO4, respectively [28]; these enzymes have a crucial role in the RNAi process. 
As peanut seeds mature, multiple changes in their physiology and chemical composition occur $[41,42]$, even total levels of RNA steadily increase during the first eight days of germination [43]. The sugar content in kernels changes as they mature (higher in immature

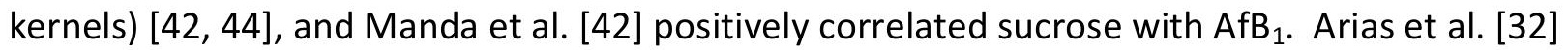
furthermore demonstrated a correlation between low water potential, laccase production by $A$. flavus, and the difference in aflatoxin accumulation in immature versus mature seeds. Hence, to gain information on small RNAs associated with reduction of aflatoxin accumulation in seeds at different maturity stages, we sequenced sRNAs in non-inoculated immature and mature seed of transformed and control peanut. This study is novel in its identification of miRNAs that were only expressed in the transformed lines, as well as miRNAs that were exclusively differentially expressed either in immature or in mature seeds. Since peanut has an indeterminate growth habit, and seeds of varying maturity stages are present at harvest information on maturityspecific or transgene-specific miRNAs could be useful to design additional targets for RNAimediated silencing.

Expression of the RNAi-5x insert also varied with seed maturity (Fig. 1). Three of the five fragments of the RNAi-5x insert (aflep, afIS/ afIJ, and afIC/ pksA) were expressed in immature seeds after $24 \mathrm{~h}$ incubation, with up to 150 -fold higher expression of fragment af/S in immature seeds of line 288-10 than in immature seeds of the control line 288-9; in mature seeds, this fragment was barely detected. Expression under the control of the 35S-promoter is not always uniform; it can be affected by environmental conditions [45], type of tissue, and developmental stage $[46,47]$. At the same time, in the pathway of RNAi, the rate of mRNA decay and the rate of siRNA decay can vary significantly [48]. Whether absence of expression at $48 \mathrm{~h}$ was due to 
low 35S-promoter driven transcription, or to fast degradation of the RNAi-5x insert by DICER proteins (DCLs) remains to be answered. It will be useful to correlate levels of mRNA/ siRNA expression/ decay and aflatoxin accumulation to determine the thresholds for efficacy.

The RNAi-5x insert used to transform peanut was developed from exogenous $A$. flavus genes, thus we expected the transgene-derived siRNAs to be among the reads that did not map to miRBase or the A. ipaënsis and A. duranensis genomes. Similar to Zhao and Song [49], who used a modified sequence of their transgene as a reference map, we used the RNAi-5x insert and the $A$. flavus genome as references to map the unmapped reads. Based on the UPLC analyses that showed high reductions in aflatoxin accumulation in the transformed lines compared to the control, we anticipated a large population of reads in high abundance that mapped to the RNAi-5x insert. In our data, we found two reads mapping the RNAi-5x insert, in very low abundance, yet, we found consistently low levels of aflatoxin in repeated challenging experiments in one or both transformed lines, particularly in line 288-10. Low copy numbers of sRNAs in peanut has been reported previously [36, 37]; where, similar to our study, the majority of the peanut miRNAs had only one copy. Low copy numbers for transgene-derived siRNAs has been reported previously as well: Jahan et al. [50] found few and low copy numbers of transgene-derived siRNAs in transgenic potato expressing a hairpin RNA construct targeting the G protein ß-subunit (PiGB1) of Phytophthora infestans, which is an essential gene involved in the pathogenicity of this pathogen. Their transgenic line had reduced DNA content and growth of the pathogen, had less disease, and had reduced transcript levels of the PiGB1 gene compared to the wildtype, nevertheless, the Illumina sRNA sequencing of the line expressing the hairpin PiGB1 revealed only nine sRNAs that mapped to the PiGB1 gene, in low abundance. 
In general, low-count miRNAs are considered to have weak or low biological importance or mRNA degradation effect [51], because these low-count miRNAs have a quick turnover in evolution [52]. In our study sRNA turnover or degradation may better explain why few and low abundance transgene-derived reads were obtained in the libraries of the transformed peanut lines. In plants, the protein HEN1 plays an important role in stabilizing miRNAs and siRNAs by methylating their $3^{\prime}$ ends $[53,54]$. This methylation protects the sRNAs against exonucleolytic shortening and/ or nontemplated nucleotide addition $[53,54]$. For example, Ramachandran and Chen [55] found reduced levels of miRNAs and siRNAs in hen1 Arabidopsis mutants, as well as miRNAs with nontemplated $3^{\prime}$ terminal nucleotides and $3^{\prime}$ end truncated miRNAs. In our data, we identified two siRNAs mapping the RNAi-5x insert, in very low abundance, but when relaxing the parameters (e.g. by slightly increasing the numbers of mismatches) the numbers of sRNAs that mapped to the RNAi-5x insert increased tremendously, with the mismatches primarily at the terminal ends of the reads (data not shown). It is therefore possible that the sRNA levels necessary for detection may not have been reached because the sRNAs were already in the degradation process at the time of sampling.

In conclusion, we obtained 89 known miRNAs, two RNAi-5x-derived siRNAs, and we predicted 10 novel miRNAs that are putatively involved in the reduction of aflatoxin accumulation. This information enhances our understanding of RNAi of aflatoxin production, and will enable the possible improvement of the RNAi technology for the control of aflatoxins.

\section{Conflicts of interest}

The authors declare not having any conflict of interest. 


\section{Acknowledgement}

This work received the financial support of USDA-ARS CRIS project 6604-21000-004-00D USAID Feed-the-Future program. We thank Monica Wang, LaTanya Johnson, Samuel Hilton, Kathy Gray, Dr. Kouassi Ayikoe, and Dr. Charles Chen for their technical assistance. Mention of trade names or commercial products in this article is solely for the purpose of providing specific information and does not imply recommendation or endorsement by the US Department of Agriculture.

\section{References}

[1] M.C. Lamb, D.A. Sternitzke, Cost of Aflatoxin to the farmer, buying point, and sheller segments of the Southeast United States peanut industry, Peanut Science, 28 (2001) 59-63.

[2] A.C. Pier, R.E. Fichtner, S.J. Cysewski, Effects of aflatoxin on the cellular immune system, Annales de la Nutrition et de l'alimentation, 31 (1977) 781-788.

[3] Y. Gong, S. Egal, A. Hounsa, P. Turner, A. Hall, K. Cardwell, C. Wild, Determinants of aflatoxin exposure in young children from Benin and Togo, West Africa: the critical role of weaning, International Journal of Epidemiology, 32 (2003) 556-562.

[4] J.H. Williams, T.D. Phillips, P.E. Jolly, J.K. Stiles, C.M. Jolly, D. Aggarwal, Human aflatoxicosis in developing countries: a review of toxicology, exposure, potential health consequences, and interventions, The American Journal of Clinical Nutrition, 80 (2004) 1106-1122.

[5] E. Azziz-Baumgartner, K. Lindblade, K. Gieseker, H.S. Rogers, S. Kieszak, H. Njapau, R. Schleicher, L.F. McCoy, A. Misore, K. DeCock, C. Rubin, L. Slutsker, G. Aflatoxin Investigative, Case-control study of an acute aflatoxicosis outbreak, Kenya, 2004, Environmental Health Perspectives, 113 (2005) 1779-1783. 
[6] M.S. Lye, A.A. Ghazali, J. Mohan, N. Alwin, R.C. Nair, An outbreak of acute hepatic encephalopathy due to severe aflatoxicosis in Malaysia, The American Journal of Tropical Medicine and Hygiene, 53 (1995) 68-72.

[7] K.G. Murugavel, P.P. Naranatt, E.M. Shankar, S. Mathews, K. Raghuram, P. Rajasambandam, V. Jayanthi, R. Surendran, A. Murali, U. Srinivas, K.R. Palaniswamy, D. Srikumari, S.P. Thyagarajan, Prevalence of aflatoxin B1 in liver biopsies of proven hepatocellular carcinoma in India determined by an in-house immunoperoxidase test, Journal of Medical Microbiology, 56 (2007) 1455-1459.

[8] J.S. Wang, T. Huang, J. Su, F. Liang, Z. Wei, Y. Liang, H. Luo, S.Y. Kuang, G.S. Qian, G. Sun, X. He, T.W. Kensler, J.D. Groopman, Hepatocellular carcinoma and aflatoxin exposure in Zhuqing Village, Fusui County, People's Republic of China, Cancer epidemiology, biomarkers \& prevention : a publication of the American Association for Cancer Research, co-sponsored by the American Society of Preventive Oncology, 10 (2001) 143-146.

[9] R. Rasooly, B. Hernlem, X. He, M. Friedman, Non-Linear relationships between aflatoxin b(1) levels and the biological response of monkey kidney vero cells, Toxins, 5 (2013) 1447-1461.

[10] R.J. Cole, J.W. Dorner, C.C. Holbrook, Advances in mycotoxin elimination and resistance., in: H.E. Pattee, H.T. Stalker (Eds.) Advances in Peanut Science, Stillwater, OK, USA, 1995, pp. 456-474.

[11] P.J. Cotty, D. Bhatnagar, Variability among atoxigenic Aspergillus flavus strains in ability to prevent aflatoxin contamination and production of aflatoxin biosynthetic pathway enzymes, Applied and Environmental Microbiology, 60 (1994) 2248-2251. 
[12] J.W. Dorner, R.J. Cole, D.T. Wicklow, Aflatoxin reduction in corn through field application of competitive fungi, Journal of Food Protection, 62 (1999) 650-656.

[13] B. Guo, J. Yu, C.C. Holbrook, T.E. Cleveland, W.C. Nierman, B.T. Scully, Strategies in Prevention of preharvest aflatoxin contamination in peanuts: aflatoxin biosynthesis, genetics and genomics, Peanut Science, 36 (2009) 11-20.

[14] A. Fire, S. Xu, M.K. Montgomery, S.A. Kostas, S.E. Driver, C.C. Mello, Potent and specific genetic interference by double-stranded RNA in Caenorhabditis elegans, Nature, 391 (1998) 806-811.

[15] D. Nowara, A. Gay, C. Lacomme, J. Shaw, C. Ridout, D. Douchkov, G. Hensel, J. Kumlehn, P. Schweizer, HIGS: host-induced gene silencing in the obligate biotrophic fungal pathogen Blumeria graminis, Plant Cell, 22 (2010) 3130-3141.

[16] A.A. Tomilov, N.B. Tomilova, T. Wroblewski, R. Michelmore, J.I. Yoder, Trans-specific gene silencing between host and parasitic plants, Plant J, 56 (2008) 389-397.

[17] C. Yin, J.E. Jurgenson, S.H. Hulbert, Development of a host-induced RNAi system in the wheat stripe rust fungus Puccinia striiformis f. sp. tritici, Molecular Plant-Microbe Interactions: MPMI, 24 (2011) 554-561.

[18] A. Frizzi, S. Huang, Tapping RNA silencing pathways for plant biotechnology, Plant Biotechnology Journal, 8 (2010) 655-677.

[19] N. Agrawal, P.V. Dasaradhi, A. Mohmmed, P. Malhotra, R.K. Bhatnagar, S.K. Mukherjee, RNA interference: biology, mechanism, and applications, Microbiology and Molecular Biology Reviews: MMBR, 67 (2003) 657-685. 
[20] E. Bernstein, A.A. Caudy, S.M. Hammond, G.J. Hannon, Role for a bidentate ribonuclease in the initiation step of RNA interference, Nature, 409 (2001) 363-366.

[21] A. Koch, K.-H. Kogel, New wind in the sails: improving the agronomic value of crop plants through RNAi-mediated gene silencing, Plant Biotechnology Journal, 12 (2014) 821-831.

[22] A.L.V. Gomes, A.M. Silva, M.T. Cordeiro, G.F. Guimarães, E.T.A. Marques Jr, F.G.C. Abath, Single-tube nested PCR using immobilized internal primers for the identification of dengue virus serotypes, Journal of Virology Methods, 145 (1) 76-79.

[23] R.S. Arias, P.M. Dang, V.S. Sobolev, RNAi-mediated Control of aflatoxins in peanut: method to analyze mycotoxin production and transgene expression in the peanut/Aspergillus pathosystem, Journal of Visualized Experiments: JoVE, (2015).

[24] J.O. Masanga, J.M. Matheka, R.A. Omer, S.C. Ommeh, E.O. Monda, A.E. Alakonya, Downregulation of transcription factor afIR in Aspergillus flavus confers reduction to aflatoxin accumulation in transgenic maize with alteration of host plant architecture, Plant Cell Reports, 34 (2015) 1379-1387.

[25] D.P. Bartel, MicroRNAs: genomics, biogenesis, mechanism, and function, Cell, 116 (2004).

[26] S.W. Ding, H. Li, R. Lu, F. Li, W.X. Li, RNA silencing: a conserved antiviral immunity of plants and animals, Virus Research, 102 (2004) 109-115.

[27] L. Matthew, RNAi for plant functional genomics, Comparative and Functional Genomics, 5 (2004) 240-244.

[28] S. Mi, T. Cai, Y. Hu, Y. Chen, E. Hodges, F. Ni, L. Wu, S. Li, H. Zhou, C. Long, S. Chen, G.J. Hannon, Y. Qi, Sorting of small RNAs into Arabidopsis argonaute complexes is directed by the $5^{\prime}$ terminal nucleotide, Cell, 133 (2008) 116-127. 
[29] S. Amaike, N.P. Keller, Aspergillus flavus, Annual Review of Phytopathology, 49 (2011) 107133.

[30] E.J. Williams, J.S. Drexler, A non-destructive method for determining peanut pod maturity, Peanut Science, 8 (1981) 134-141.

[31] P.M. Dang, C.Y. Chen, C.C. Holbrook, Evaluation of five peanut (Arachis hypogaea) genotypes to identify drought responsive mechanisms utilising candidate-gene approach, Functional Plant Biology, 40 (2013) 1323-1333.

[32] R.S. Arias, V.S. Sobolev, V.A. Orner, P.M. Dang, M.C. Lamb, Potential involvement of Aspergillus flavus laccases in peanut invasion at low water potential, Plant Pathology, 63 (2014) 354-364.

[33] V.S. Sobolev, J.W. Dorner, Cleanup procedure for determination of aflatoxins in major agricultural commodities by liquid chromatography, Journal of AOAC International, 85 (2002) 642-645.

[34] S. Biselli, C. Hummert, Development of a multicomponent method for Fusarium toxins using LC-MS/MS and its application during a survey for the content of T-2 toxin and deoxynivalenol in various feed and food samples, Food additives and contaminants, 22 (2005) 752-760.

[35] X. Dai, P.X. Zhao, psRNATarget: a plant small RNA target analysis server, Nucleic Acids Res, (2011).

[36] X. Chi, Q. Yang, X. Chen, J. Wang, L. Pan, M. Chen, Z. Yang, Y. He, X. Liang, S. Yu, Identification and characterization of microRNAs from peanut (Arachis hypogaea L.) by high-throughput sequencing, PLoS One, 6 (2011) e27530. 
[37] C.Z. Zhao, H. Xia, T.P. Frazier, Y.Y. Yao, Y.P. Bi, A.Q. Li, M.J. Li, C.S. Li, B.H. Zhang, X.J. Wang, Deep sequencing identifies novel and conserved microRNAs in peanuts (Arachis hypogaea L.), BMC Plant Biology, 10 (2010) 3.

[38] M.L. Tinoco, B.B. Dias, R.C. Dall'Astta, J.A. Pamphile, F.J. Aragao, In vivo trans-specific gene silencing in fungal cells by in planta expression of a double-stranded RNA, BMC Biology, 8 (2010) 27.

[39] K.M. McGinnis, RNAi for functional genomics in plants, Briefings in Functional Genomics, 9 (2010) 111-117.

[40] A. Vermeulen, L. Behlen, A. Reynolds, A. Wolfson, W.S. Marshall, J.O.N. Karpilow, A. Khvorova, The contributions of dsRNA structure to Dicer specificity and efficiency, RNA, 11 (2005) 674-682.

[41] S.M.M. Basha, J.P. Cherry, C.T. Young, Changes in free amino acids, carbohydrates, and proteins of maturing seeds from various peanut (Arachis hypogaea L.) Cultivars, Cereal Chemistry, 53 (1976) 586-596.

[42] A. Manda, P.N. Bodapati, N.C. Rachaputi, G. Wright, S. Fukai, Aflatoxins and their relationship with sugars in peanut (Arachis hypogaea L.). in: 4th International Crop Science Congress, The Regional Institute Ltd, Brisbane, Australia, 2004.

[43] J.H. Cherry, Nucleic acid, mitochondria, \& enzyme changes in cotyledons of peanut seeds during germination, Plant Physiol, 38 (1963) 440-446.

[44] S.S. Uppala, Factors affecting pre-harvest aflatoxin contamination of peanut (Arachis hypogaea L.), in, Ph.D. dissertation, Auburn University, 2011. 
[45] F. Maghuly, M.A. Khan, E.B. Fernandez, P. Druart, B. Watillon, M. Laimer, Stress regulated expression of the GUS-marker gene (uidA) under the control of plant calmodulin and viral 35S promoters in a model fruit tree rootstock: Prunus incisa $\mathrm{x}$ serrula, Journal of biotechnology, 135 (2008) 105-116.

[46] M.C. de Mesa, N. Santiago-Domenech, F. Pliego-Alfaro, M.A. Quesada, J.A. Mercado, The CaMV 35 s promoter is highly active on floral organs and pollen of transgenic strawberry plants, Plant Cell Reports, 23 (2004) 32-38.

[47] G. Sunilkumar, L. Mohr, E. Lopata-Finch, C. Emani, K.S. Rathore, Developmental and tissuespecific expression of CaMV 35 S promoter in cotton as revealed by GFP, Plant Mol Biol, 50 (2002) 463-479.

[48] M.A.C. Groenenboom, A.F.M. Marée, P. Hogeweg, The RNA silencing pathway: the bits and pieces that matter, PLoS Comput Biol, 1 (2005) e21.

[49] D. Zhao, G.Q. Song, High-throughput sequencing as an effective approach in profiling small RNAs derived from a hairpin RNA expression vector in woody plants, Plant science : an International Journal of Experimental Plant Biology, 228 (2014) 39-47.

[50] S.N. Jahan, A.K. Asman, P. Corcoran, J. Fogelqvist, R.R. Vetukuri, C. Dixelius, Plant-mediated gene silencing restricts growth of the potato late blight pathogen Phytophthora infestans, Journal of Experimental Botany, 66 (2015) 2785-2794.

[51] M. Hackenberg, P. Gustafson, P. Langridge, B.-J. Shi, Differential expression of microRNAs and other small RNAs in barley between water and drought conditions, Plant Biotechnology Journal, 13 (2015) 2-13. 
[52] H. Liang, W.H. Li, Lowly expressed human microRNA genes evolve rapidly, Molecular Biology and Evolution, 26 (2009) 1195-1198.

[53] M. Sanei, X. Chen, Mechanisms of microRNA turnover, Curr Opin Plant Biol, 27 (2015) 199206.

[54] Z. Zhang, Y.W. Qin, G. Brewer, Q. Jing, MicroRNA degradation and turnover: regulating the regulators, Wiley interdisciplinary reviews. RNA, 3 (2012) 593-600.

[55] V. Ramachandran, X. Chen, Degradation of microRNAs by a family of exoribonucleases in Arabidopsis, Science, 321 (2008) 1490-1492. 


\section{Figure legends}

Fig. 1. Detection and expression of selectable marker NPTII and the RNAi-5x-insert. A: Singletube-nested-PCR detection of NPTII. M: 1kb plus DNA ladder (Invitrogen, MA); MM: PCR master mix (negative control), pDNA: plasmid DNA of Agrobacterium harboring plasmid p5xCAPD that contained the RNAi-5x-insert (positive control). The $500 \mathrm{bp}$ band represented the presence of NPTII. B: PCR detection of the RNAi-5x-insert. M: 1kb plus DNA ladder (Invitrogen, MA); AB: Agrobacterium harboring plasmid p5xCAPD that contained the RNAi-5x-insert (positive controls); PT: Plant Tissue. Fractions $1 / 2$ and $1 / 10$ represent 2 - and 10 -fold dilutions of DNA. The 938 bp band represented the presence of the RNAi-5x-insert. C: Real-Time PCR detection of expression of the RNAi-5x-insert using primers sets RT_5X_1 and RT_5x_2, on immature and mature cotyledons of transformed lines $288-10$ and $288-11$ at 24 and $48 \mathrm{hr}$ incubation; gray line: $C_{T}=1$. Histograms represent the means and standard error bars $(T)$ of three biological samples with three technical replicates. The relative quantification of RNAi insert was normalized with respect to the housekeeping gene actin as an internal control and comparative fold expression of the transgene.

Fig. 2. Aflatoxins $B_{1}$ and $B_{2}$ in half peanut cotyledons after $72 \mathrm{~h}$ incubation with Aspergillus flavus. Control: seeds of 288-9 non-transgenic line; RNAi: combined seeds from transformed lines 288-10 and 288-11 to silence five aflatoxin-synthesis genes. Mean values with the corresponding standard error bars $(\mathrm{T})$ of repeated biological samples are represented. The level of $\mathrm{AfB}_{1}$ and $\mathrm{AfB}_{2}$ after $72 \mathrm{~h}$ incubation was $92 \%(P<0.009)$ and $95 \%(P<0.001)$ lower than the control, respectively. 
Fig. 3. Aflatoxins $B_{1}$ and $B_{2}$ in half peanut cotyledons after 24,48 , and $72 \mathrm{~h}$ incubation with Aspergillus flavus. Control: seeds of 288-9 non-transgenic line; RNAi 288-10 and RNAi 288-11: seeds from transformed lines 288-10 and 288-11, respectively. Immature and mature seeds were analyzed separately. Mean values with the corresponding standard error bars (T) of triplicate biological samples are represented. A: Aflatoxin $B_{1}$ in mature seed of control line (288-9I); B: Aflatoxin $B_{1}$ in immature seed of control line (288-9M); C: Aflatoxin $B_{2}$ in mature seed of transformed line (288-10I); D: Aflatoxin $B_{2}$ in immature seed of transformed line (28810M). In mature seeds of line 288-10, the levels of $A B_{1}$ (Fig. 3 A) and $A f B_{2}$ (Fig. 3 C) were $80 \%$ and $78 \%$ lower, respectively, compared to the control. In immature seed the reduction in the level of $\mathrm{AfB}_{1}$ at 48 and $72 \mathrm{~h}$ was $100 \%$ and $100 \%$ for line $288-10(P<0.004)$, and $98 \%$ and $98 \%(P$ $<0.002$ ) for line $288-11$, respectively.

Fig. 4. Length distribution (15- $45 \mathrm{nt}$ ) of mappable small RNAs in libraries of non-inoculated immature (I) and mature (M) seeds of control line 288-9, and transformed lines 288-10 and 28811, after 24 and $48 \mathrm{~h}$ incubation. A: Immature seed of control line (288-9l); B: Mature seed of control line (288-9M); C: Immature seed of transformed line (288-10I); D: Mature seed of transformed line (288-10M); E: Immature seed of transformed line (288-11I); F: Mature seed of transformed line (288-11M).

Fig. 5. Heat map of 89 differentially expressed known miRNAs in peanut of non-inoculated immature (I) and mature (M) seeds of transformed lines 288-10 and 288-11, compared to the control line 288-9, after 24 and $48 \mathrm{~h}$ incubation. 
Fig. 6. Heat map of 10 differentially expressed novel peanut miRNAs of non-inoculated immature (I) and mature (M) seeds of transformed lines 288-10 and 288-11, compared to the control line 288-9, after 24 and $48 \mathrm{~h}$ incubation.

\section{Table legends}

Table 1. Differentially expressed predicted novel miRNAs in immature (I) and mature (M) seeds of transformed lines 288-10 and 288-11, compared to the control 288-9, expressing the RNAi-5x insert that targets five genes involved in aflatoxin production.

Table 2. Small RNAs complementary to the RNAi-5x insert in libraries of non- inoculated immature (I) and mature (M) seeds of control line 288-9 and transformed lines 288-10 and 28811 , after 24 and $48 \mathrm{~h}$ incubation.

Table 3. Small RNAs in libraries of immature (I) and mature (M) seeds of control line 288-9 and transformed lines 288-10 and 288-11 that map to the genome of $A$. flavus.

Table A. 1. Total abundance of known miRNA families identified from non-inoculated immature (I) and mature (M) seeds of control line 288-9 and transformed lines 288-10 and 288-11, after 24 and $48 \mathrm{~h}$ incubation.

Table A. 2. Abundance of known miRNAs identified from non-inoculated immature (I) and mature (M) seeds of control line 288-9 and transformed lines 288-10 and 288-11, after 24 and $48 \mathrm{~h}$ incubation.

Table A. 3. Differentially expressed known miRNAs in immature (I) and mature (M) seeds of transformed lines 288-10 (10) and 288-11 (11), compared to the control 288-9 (9), expressing the RNAi-5x insert that targets five genes involved in aflatoxin production. 
Table A. 4. Abundance of putatively novel miRNAs in non-inoculated immature (I) and mature (M) seeds of control line 288-9 and transformed lines 288-10 and 288-11, after 24 and 48 h incubation.

Table A. 5. Predicted targets of putatively novel miRNAs in seeds of transformed peanut lines 288-10 and 288-11, expressing the RNAi-5x insert that targets five genes involved in aflatoxin production, by psRNAtarget. 
Table 1. Differentially expressed predicted novel miRNAs in immature (I) and mature (M) seeds of transformed lines $288-10$ and

288-11, compared to the control 288-9, expressing the RNAi-5x insert that targets five genes involved in aflatoxin production.

\begin{tabular}{|c|c|c|c|c|c|c|c|c|c|c|}
\hline \multirow[b]{2}{*}{ miRNA name } & \multirow[b]{2}{*}{ miRNA sequence } & \multirow[b]{2}{*}{ Length } & \multicolumn{8}{|l|}{$\log 2$} \\
\hline & & & $\begin{array}{c}288- \\
10 \mathrm{l}-24 \mathrm{~h}\end{array}$ & $\begin{array}{c}288- \\
10 \mathrm{l}-48 \mathrm{~h}\end{array}$ & $\begin{array}{c}288- \\
10 \mathrm{M}- \\
24 \mathrm{~h} \\
\end{array}$ & $\begin{array}{c}288- \\
10 \mathrm{M}- \\
48 \mathrm{~h} \\
\end{array}$ & $\begin{array}{c}288- \\
111-24 h\end{array}$ & $\begin{array}{c}288- \\
111-48 h\end{array}$ & $\begin{array}{c}288- \\
11 \mathrm{M}- \\
24 \mathrm{~h}\end{array}$ & $\begin{array}{c}288- \\
11 \mathrm{M}- \\
48 \mathrm{~h}\end{array}$ \\
\hline ahy-novel-1 & UAGACAGAGAAUGCAAAGACU & 21 & 2.19 & -2.21 & 0.97 & -1.89 & -0.70 & -2.23 & -0.39 & -2.41 \\
\hline ahy-novel-2 & GGACGAGGACCAGGAAGACCU & 21 & 5.26 & -1.24 & 0.55 & -3.95 & 0.05 & -1.44 & -0.42 & -5.41 \\
\hline ahy-novel-3 & CAAAGACUGAACCACAUUUGG & 21 & 2.66 & -2.30 & 0.25 & -2.37 & -0.62 & -2.29 & -0.95 & -2.44 \\
\hline ahy-novel-4 & UAACUUUGAAUAUCCGGAGAA & 21 & 4.97 & -4.36 & 2.94 & -4.23 & -0.13 & -5.18 & 0.93 & -4.43 \\
\hline ahy-novel-5 & GGAAGUCGCCGAGUGACUCGG & 21 & 1.06 & -1.44 & 0.56 & -1.68 & -0.38 & -0.67 & 0.51 & -2.57 \\
\hline ahy-novel-6 & CCGAACAAGAUCGAACAAACU & 21 & 15.78 & -0.31 & 11.03 & -1.28 & 0.00 & -0.65 & 0.00 & -2.44 \\
\hline ahy-novel-7 & CCUAUUUCGGAAGAACAGCCA & 21 & 1.27 & -0.98 & 2.01 & -1.74 & 0.68 & -1.22 & 1.10 & -1.88 \\
\hline ahy-novel-8 & UGAGGAUUCUUGUUAACUUUGC & 22 & 3.11 & -3.27 & 1.04 & -3.30 & 0.41 & -3.62 & 0.15 & -2.74 \\
\hline ahy-novel-9 & GGCAAAAGUUGAAUGAACUCAAUU & 24 & 16.92 & -3.68 & 0.00 & -14.51 & 0.00 & -4.02 & 9.60 & -14.51 \\
\hline ahy-novel-10 & CCUGGACCACCGAAUCCUGUGCCU & 24 & 1.25 & 2.14 & 1.31 & -0.25 & 1.24 & 0.46 & 0.93 & -0.63 \\
\hline
\end{tabular}


Table 2. Small RNAs complementary to the RNAi-5x insert in libraries of non- inoculated immature (I) and mature (M) seeds of control line 288-9 and transformed lines 288-10 and 288-11, after 24 and $48 \mathrm{~h}$ incubation.

\begin{tabular}{|c|c|c|c|c|c|c|c|c|c|c|c|c|c|c|}
\hline siRNA name & Target & siRNA sequence & $\begin{array}{c}288- \\
91- \\
24 \mathrm{~h} \\
\end{array}$ & $\begin{array}{c}288- \\
91- \\
48 \mathrm{~h} \\
\end{array}$ & $\begin{array}{l}288- \\
9 \mathrm{M}- \\
24 \mathrm{~h}\end{array}$ & $\begin{array}{l}288- \\
9 \mathrm{M}- \\
48 \mathrm{~h}\end{array}$ & $\begin{array}{l}288- \\
101- \\
24 \mathrm{~h}\end{array}$ & $\begin{array}{l}288- \\
10 \mathrm{I}- \\
48 \mathrm{~h}\end{array}$ & $\begin{array}{c}288- \\
10 \mathrm{M}- \\
24 \mathrm{~h}\end{array}$ & $\begin{array}{c}288- \\
10 \mathrm{M}- \\
48 \mathrm{~h}\end{array}$ & $\begin{array}{l}288- \\
11 /- \\
24 \mathrm{~h}\end{array}$ & $\begin{array}{l}288- \\
11 /- \\
48 \mathrm{~h}\end{array}$ & $\begin{array}{c}288- \\
11 \mathrm{M}- \\
24 \mathrm{~h}\end{array}$ & $\begin{array}{c}288- \\
11 \mathrm{M}- \\
48 \mathrm{~h}\end{array}$ \\
\hline ahy-siRNA-1 & RNAi-5x insert & GGGGGUACCCGUCUAUCAG & 0 & 1 & 0 & 0 & 1 & 0 & 0 & 1 & 0 & 0 & 0 & 0 \\
\hline ahy-siRNA-2 & RNAi-5x insert & UCGAUGCGGCACAAUC & 0 & 0 & 0 & 0 & 0 & 2 & 0 & 0 & 0 & 0 & 0 & 0 \\
\hline
\end{tabular}


Table 3. Small RNAs in libraries of immature (I) and mature (M) seeds of control line 288-9 and transformed lines 288-10 and 288-11

that map to the genome of $A$. flavus.

\begin{tabular}{|c|c|c|c|c|c|c|c|c|c|c|c|c|c|}
\hline sRNA & siRNA sequence & $\begin{array}{c}288- \\
91- \\
24 \mathrm{~h} \\
\end{array}$ & $\begin{array}{c}288- \\
91- \\
48 \mathrm{~h} \\
\end{array}$ & $\begin{array}{l}288- \\
9 \mathrm{M}- \\
24 \mathrm{~h}\end{array}$ & $\begin{array}{l}288- \\
9 \mathrm{M}- \\
48 \mathrm{~h}\end{array}$ & $\begin{array}{l}288- \\
10 \mathrm{-} \\
24 \mathrm{~h}\end{array}$ & $\begin{array}{l}288- \\
10 \mathrm{-} \\
48 \mathrm{~h} \\
\end{array}$ & $\begin{array}{c}288- \\
10 \mathrm{M}- \\
24 \mathrm{~h}\end{array}$ & $\begin{array}{c}288- \\
10 \mathrm{M}- \\
48 \mathrm{~h}\end{array}$ & $\begin{array}{l}288- \\
111- \\
24 \mathrm{~h}\end{array}$ & $\begin{array}{l}288- \\
111- \\
48 \mathrm{~h} \\
\end{array}$ & $\begin{array}{c}288- \\
11 \mathrm{M}- \\
24 \mathrm{~h}\end{array}$ & $\begin{array}{c}288- \\
11 \mathrm{M}- \\
48 \mathrm{~h}\end{array}$ \\
\hline 1089613_8 & ACAAGCGAGAAUCAUACCAC & 0 & 0 & 0 & 0 & 1 & 0 & 1 & 0 & 0 & 1 & 4 & 0 \\
\hline 3942292_2 & GGUCAAAAAAUACACCCGAA & 0 & 0 & 0 & 0 & 1 & 0 & 0 & 0 & 0 & 0 & 0 & 0 \\
\hline 3971843_2 & AGUCUGUUGUAACACAAUAG & 0 & 0 & 0 & 0 & 2 & 0 & 0 & 0 & 0 & 0 & 0 & 0 \\
\hline 5217647_2 & CUGGUACAAGGGUUGGGAGA & 0 & 0 & 0 & 0 & 0 & 1 & 0 & 1 & 0 & 0 & 0 & 0 \\
\hline 6331253_2 & UAUUAGACCCGAACCCGACC & 0 & 0 & 0 & 0 & 0 & 1 & 0 & 0 & 0 & 0 & 0 & 1 \\
\hline $7612412 \_2$ & UUAGACCCGAACCCGACCCA & 0 & 0 & 0 & 0 & 0 & 0 & 0 & 2 & 0 & 0 & 0 & 0 \\
\hline 3045591_3 & CCUACCCUACCCUACCCUACC & 0 & 0 & 0 & 0 & 0 & 1 & 0 & 0 & 0 & 1 & 1 & 0 \\
\hline 3317823_3 & UCCCGAAGUUACGGAUCCAAU & 0 & 0 & 0 & 0 & 0 & 0 & 3 & 0 & 0 & 0 & 0 & 0 \\
\hline 3396602_3 & UUGAUUUUGUGCCUCUGUUUU & 0 & 0 & 0 & 0 & 1 & 1 & 0 & 0 & 0 & 1 & 0 & 0 \\
\hline 4105308_2 & CCCUACCCUACCCUACCCUAC & 0 & 0 & 0 & 0 & 0 & 0 & 0 & 0 & 0 & 2 & 0 & 0 \\
\hline 4120167_2 & CGAAUCACCUGGAUCAGCCCG & 0 & 0 & 0 & 0 & 0 & 0 & 0 & 2 & 0 & 0 & 0 & 0 \\
\hline 5440940_2 & ACAAAUCUAGCCAAGGUCGGG & 0 & 0 & 0 & 0 & 0 & 2 & 0 & 0 & 0 & 0 & 0 & 0 \\
\hline 5910034_2 & UGGAUUGGAUUGGAUUUCGGA & 0 & 0 & 0 & 0 & 0 & 0 & 0 & 0 & 0 & 0 & 1 & 0 \\
\hline 5992160_2 & ACAAGCGAGAAUCAUACCACU & 0 & 0 & 0 & 0 & 1 & 1 & 0 & 0 & 0 & 0 & 0 & 0 \\
\hline 5992160_2 & ACAAGCGAGAAUCAUACCACU & 0 & 0 & 0 & 0 & 1 & 1 & 0 & 0 & 0 & 0 & 0 & 0 \\
\hline 7149026_2 & ACCCCUAACUUCGAUCCCAGA & 0 & 0 & 0 & 0 & 0 & 0 & 0 & 0 & 2 & 0 & 0 & 0 \\
\hline 2161448_4 & UUAUUAGACCCGAACCCGACCC & 0 & 0 & 0 & 0 & 0 & 1 & 0 & 1 & 1 & 1 & 0 & 0 \\
\hline $6288002 \_2$ & UGAGGAAGAAGAGGAAGAGUCU & 0 & 0 & 0 & 0 & 0 & 0 & 0 & 1 & 0 & 1 & 0 & 0 \\
\hline 6445204_2 & ACAAGCGAGAAUCAUACCACUA & 0 & 0 & 0 & 0 & 0 & 0 & 0 & 0 & 0 & 0 & 1 & 0 \\
\hline 5271469_2 & AGAGGAGGAGGAGGAGAAGGAGA & 0 & 0 & 0 & 0 & 1 & 0 & 0 & 0 & 0 & 0 & 1 & 0 \\
\hline 5704364_2 & UCGUUUCUCCUCCUUUUCCUCCU & 0 & 0 & 0 & 0 & 1 & 0 & 0 & 1 & 0 & 0 & 0 & 0 \\
\hline 400176_19 & GGUGGUGGUGGUGGUAGUAGUAGU & 0 & 0 & 0 & 0 & 0 & 6 & 3 & 0 & 1 & 1 & 7 & 1 \\
\hline 1406521_6 & UGGUUUGGUUCGGUUUGUUCGGUU & 0 & 0 & 0 & 0 & 0 & 2 & 1 & 0 & 1 & 1 & 0 & 0 \\
\hline 2010238_4 & CCUCCUCCUUCUCCUCUUUCUUCU & 0 & 0 & 0 & 0 & 0 & 0 & 0 & 0 & 1 & 0 & 0 & 1 \\
\hline
\end{tabular}

Power, Page 33, Plant Science 


\begin{tabular}{|c|c|}
\hline 2518648_4 & AUCUUCGUCUUCAUCCUCGUCCUC \\
\hline 4424247_2 & GAUGAUGAUGAUGAUGGAGGAGGA \\
\hline 4434044_2 & UGAUGAUGAUGAUGGAGGAGGAGA \\
\hline 4439561_2 & UCCUCUUCCUCUUCUUCUCCUUCU \\
\hline 5558673_2 & AAGAAAAGAAAAGAAAAGAGAGGG \\
\hline 5786288_2 & AGUAGAAGACGAGGAGGAGGAGGA \\
\hline 5819598_2 & GAGAGGAGAGGAGAGGAGAGGAGA \\
\hline 7372646_2 & ACAAGCGAGAAUCAUACCACUAGA \\
\hline 7390105_2 & GUGUCGUGUCGUGUCGUGUCGUGU \\
\hline 7472402_2 & AGGAGGAGGAGGAAACGAAAAAGA \\
\hline
\end{tabular}

$\begin{array}{lllllllllll}0 & 0 & 0 & 0 & 0 & 0 & 0 & 0 & 1 & 0 & 0 \\ 0 & 0 & 0 & 0 & 0 & 1 & 0 & 1 & 0 & 0 & 0 \\ 0 & 0 & 0 & 0 & 0 & 0 & 0 & 1 & 0 & 0 & 0 \\ 0 & 0 & 0 & 0 & 1 & 0 & 0 & 0 & 0 & 1 & 0 \\ 0 & 0 & 0 & 0 & 1 & 0 & 0 & 1 & 0 & 0 & 0 \\ 0 & 0 & 0 & 0 & 1 & 0 & 0 & 0 & 1 & 0 & 0 \\ 0 & 0 & 0 & 2 & 0 & 0 & 0 & 0 & 0 & 0 & 0 \\ 0 & 0 & 0 & 0 & 1 & 1 & 0 & 0 & 0 & 0 & 0 \\ 0 & 0 & 0 & 0 & 1 & 1 & 0 & 0 & 0 & 0 & 0 \\ 0 & 0 & 0 & 0 & 1 & 0 & 0 & 0 & 1 & 0 & 0\end{array}$



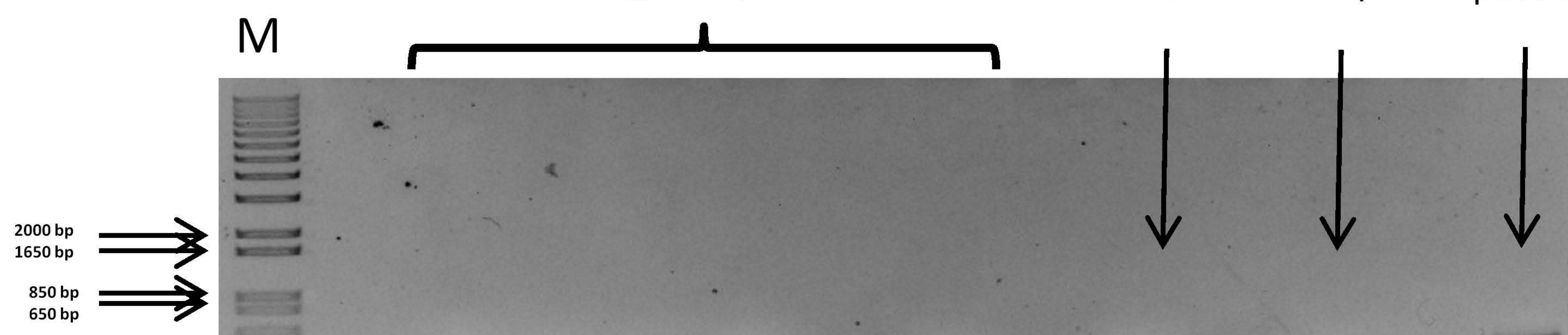

A
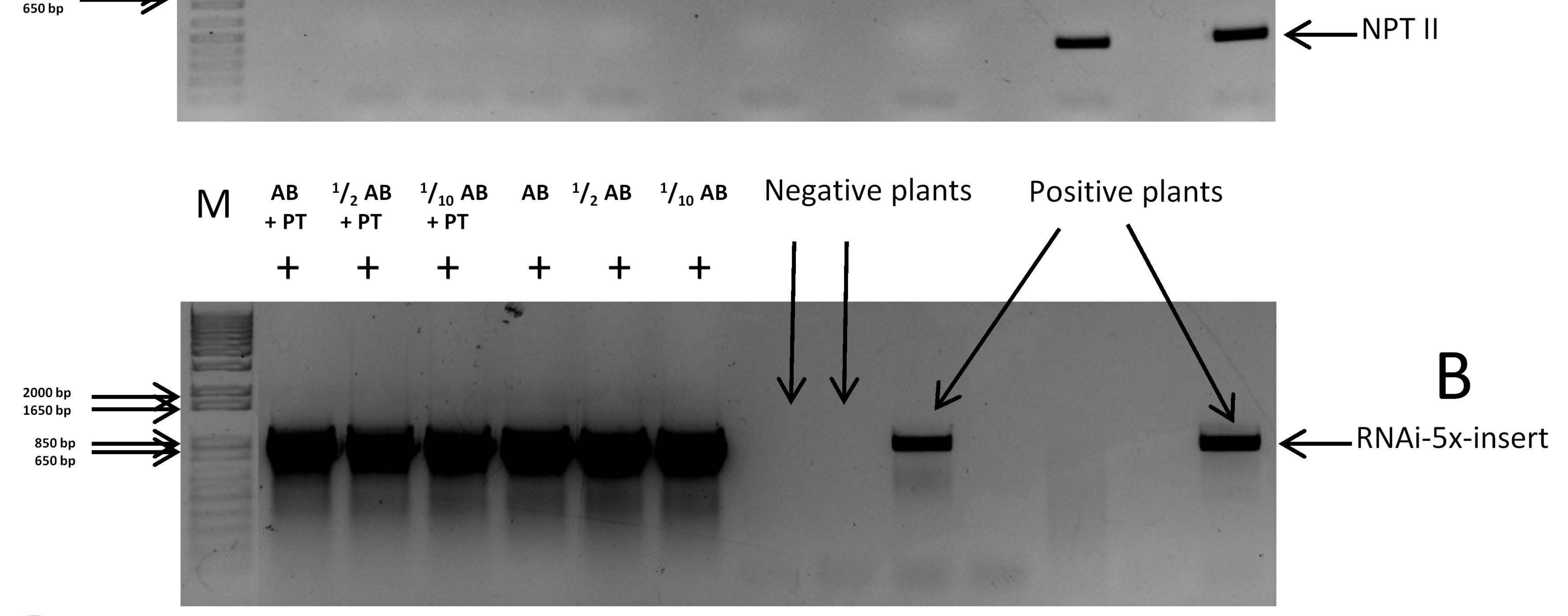

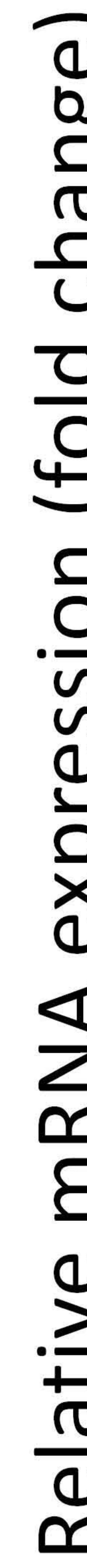

160

140

$120 y$

20

15

10

5

0
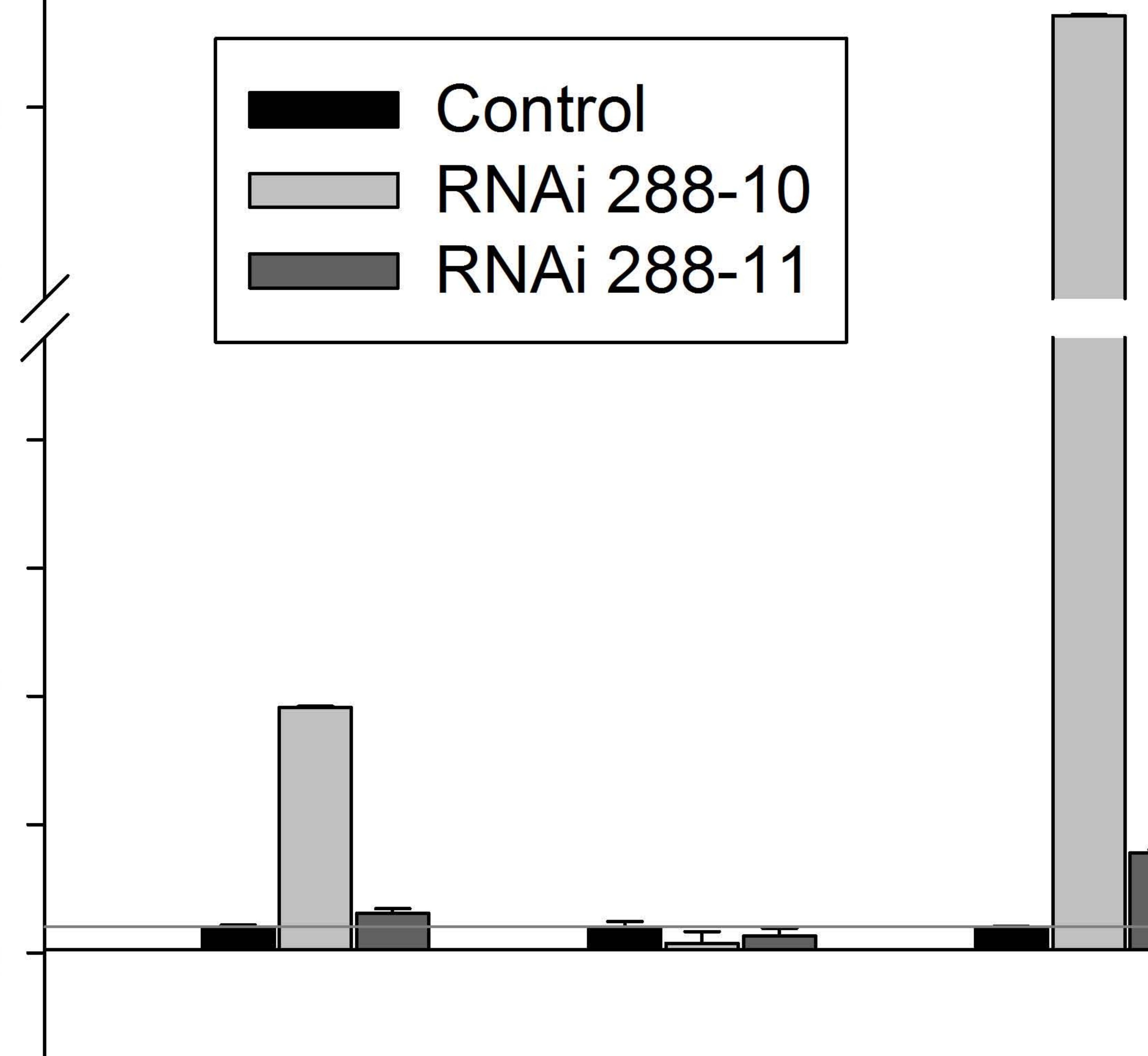

$24 \mathrm{~h}$

C

5

0

$\square_{1} \|$

Immature Mature

Immature Mature

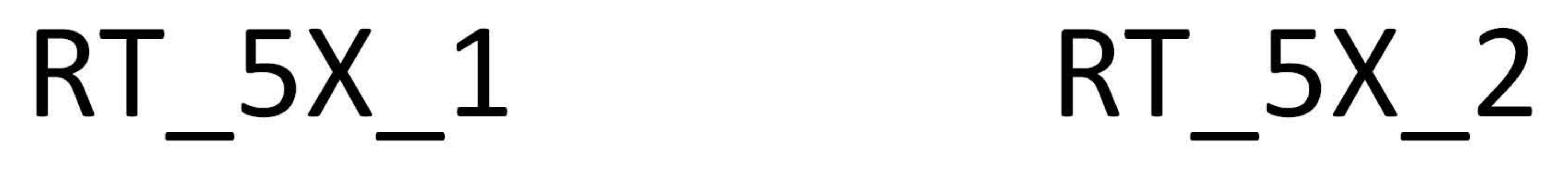




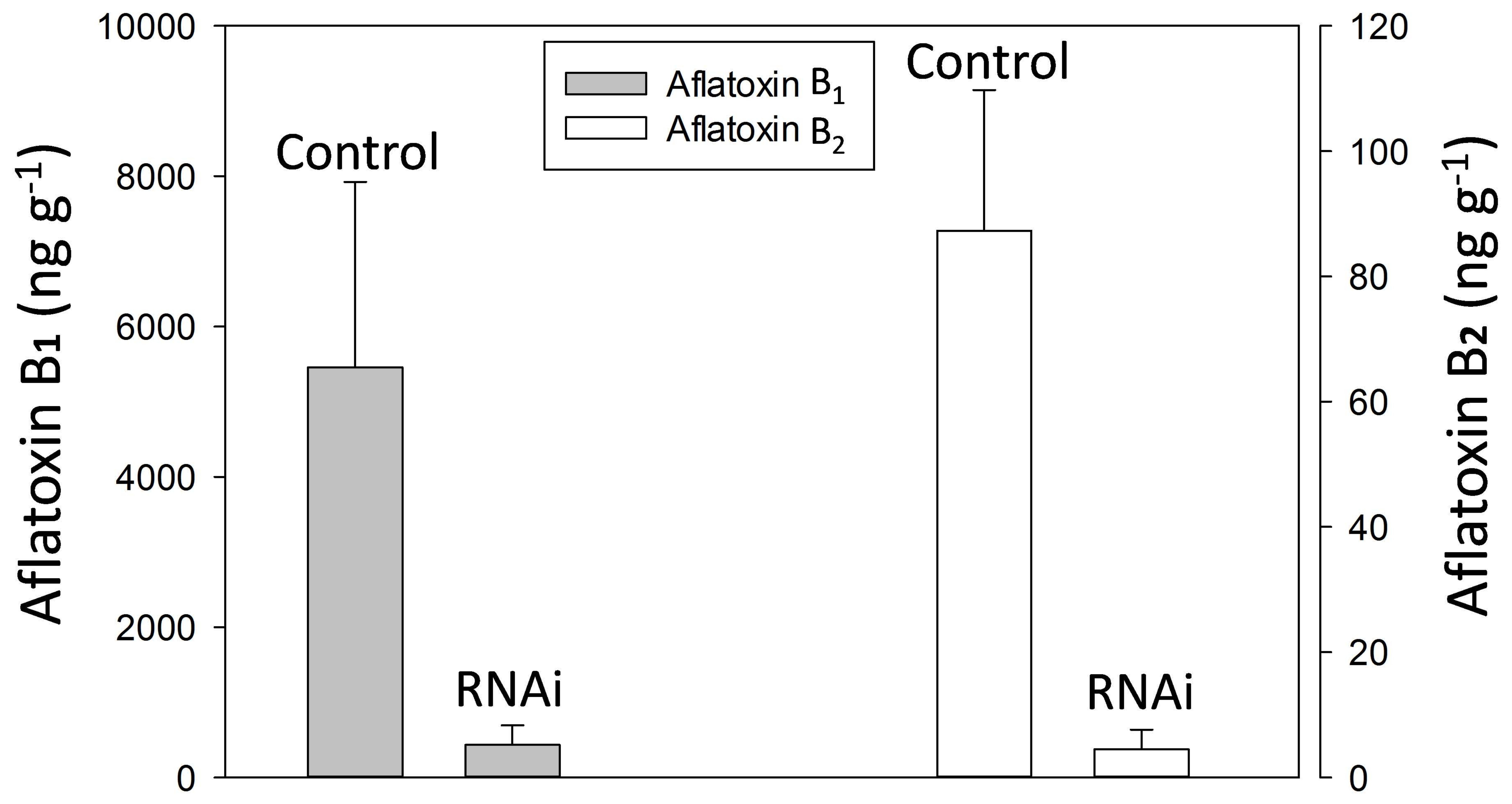




ahy-miR894y ahy-miR894x ahy-miR1507-3px ahy-miR1511y ahy-miR1511z ahy-MIR1511-p5 ahy-miR1511x ahy-miR2118a-3px ahy-miR2118a-3py ahy-MIR2592bj-p5x ahy-miR2673ax ahy-MIR2916-p5y ahy-MIR2916-p3x ahy-miR3508 ahy-MIR3509-p5x ahy-MIR3509-p3x ahy-MIR3509-p3y ahy-MIR3513-p5 ahy-MIR3513-p3 ahy-miR3514-3p ahy-miR3516x ahy-MIR3521-p3 ahy-miR5072x ahy-MIR5083-p3x ahy-MIR5368-p5x ahy-MIR5538-p5x ahy-MIR5652-p3x ahy-MIR5652-p5x ahy-MIR5652-p5y ahy-MIR5724-p3x ahy-MIR6025c-p3y ahy-MIR6108a-p3z ahy-miR6300xc ahy-miR6300xb ahy-miR6300xa ahy-miR6300z ahy-MIR6300-p5x ahy-MIR6300-p3x ahy-miR6300x ahy-MIR6300-p5x ahy-miR6478z ahy-miR6478y ahy-miR6478x ahy-miR8175x ahy-MIR9410-p3x

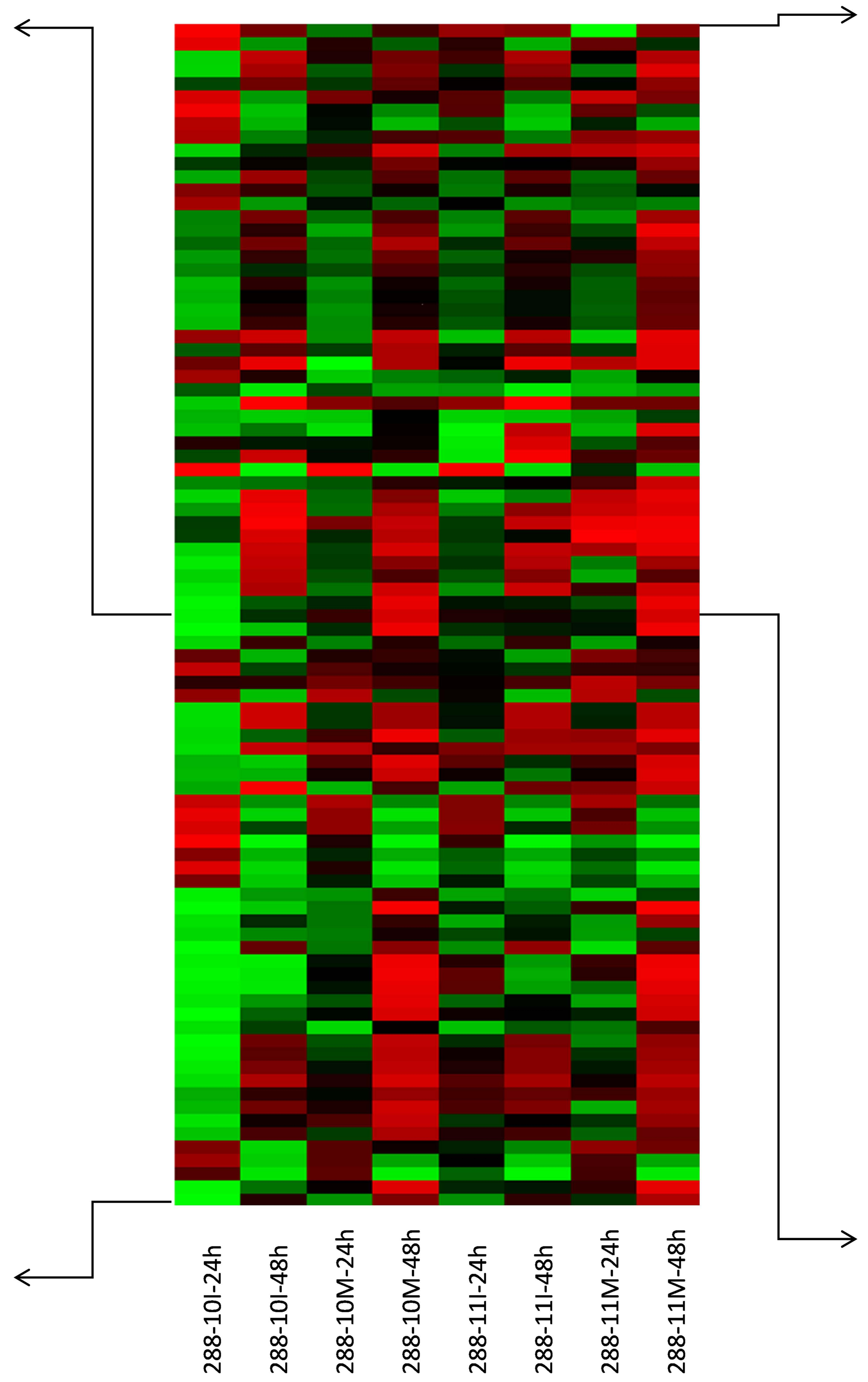

ahy-miR156fx ahy-miR156b-5p ahy-MIR156e-p3x ahy-MIR156c-p3x ahy-MIR156c-p3x ahy-miR156adx ahy-miR156ad ahy-miR156az ahy-miR156ay ahy-MIR159b-p3x ahy-MIR159-p5 ahy-miR160a-5p ahy-miR160-5p ahy-miR162ax ahy-miR166h-3pa ahy-miR166a-5py ahy-miR166a-5p ahy-miR166a-3pxb ahy-miR166a-3pxc ahy-miR166a-3py ahy-miR166a-3pz ahy-miR166a-3px ahy-miR166a-3p ahy-miR166h-5p ahy-miR166a-5px ahy-miR167e-3px ahy-miR169l-3px ahy-miR169k ahy-MIR171b-p3x ahy-MIR172i-p5x ahy-miR319a ahy-miR319gx ahy-miR319dx ahy-MIR390b-p5x ahy-miR390a-5p ahy-miR398c ahy-miR408-3px ahy-miR408-3p ahy-MIR408b-p3 ahy-miR482b-3py ahy-miR482b-3pz ahy-miR482a-3px ahy-miR482b-3px ahy-miR894z

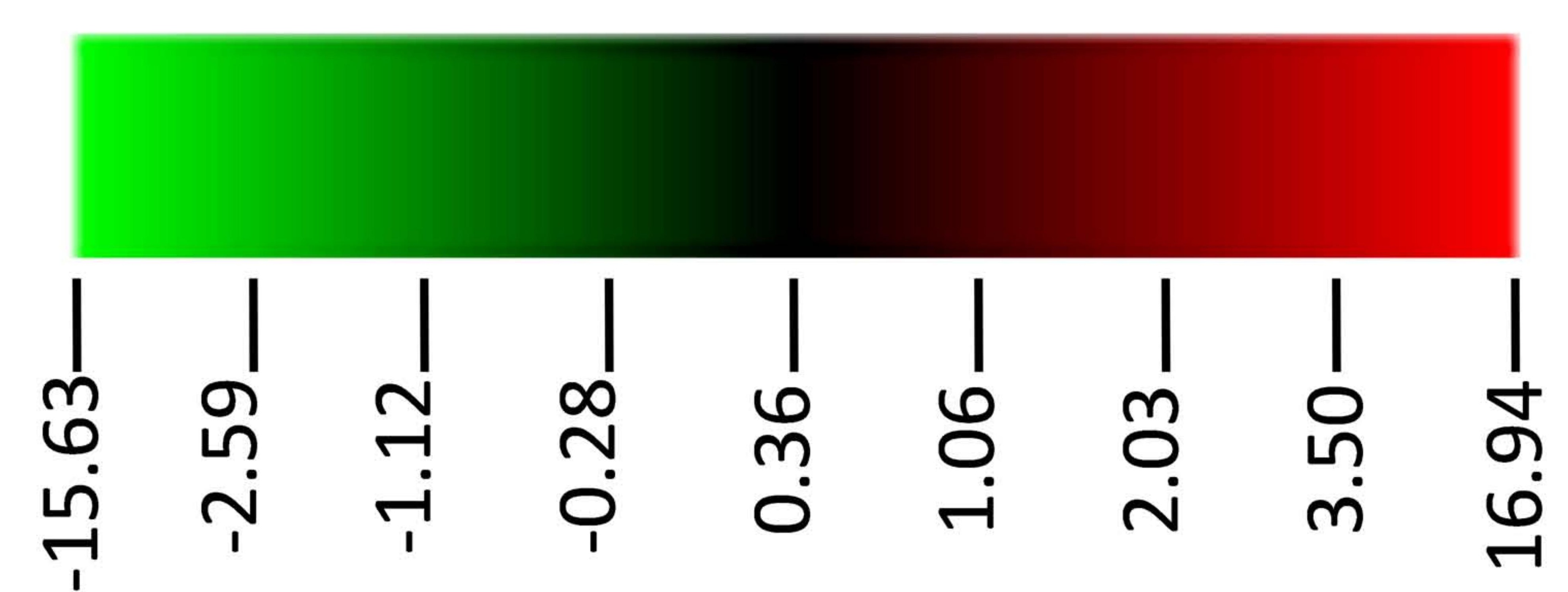


ahy-novel-1

ahy-novel-2

ahy-novel-3

ahy-novel-4

ahy-novel-5

ahy-novel-6

ahy-novel-7

ahy-novel-8

ahy-novel-9

ahy-novel-10

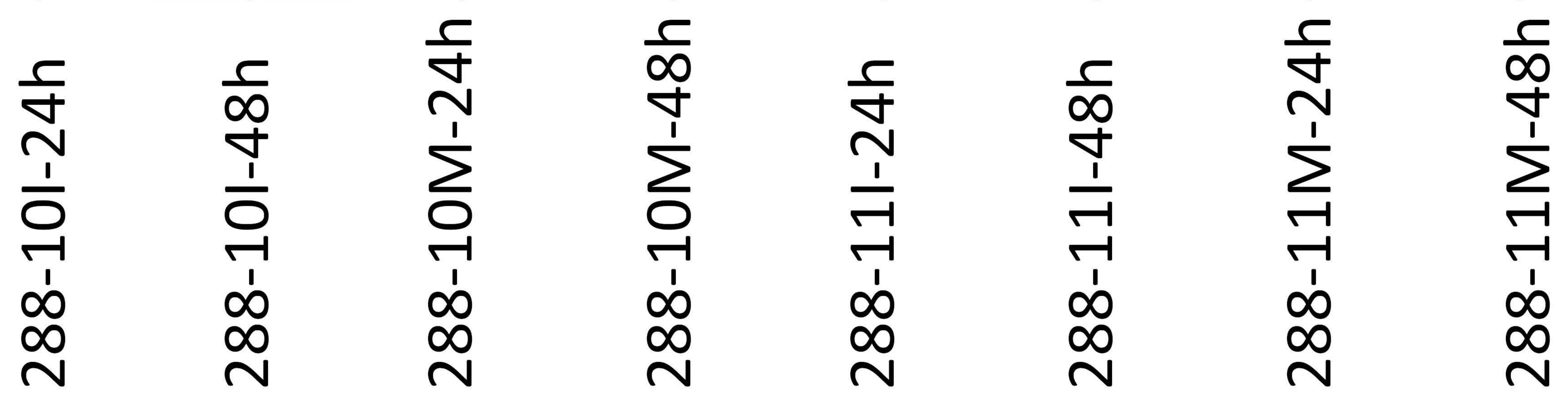

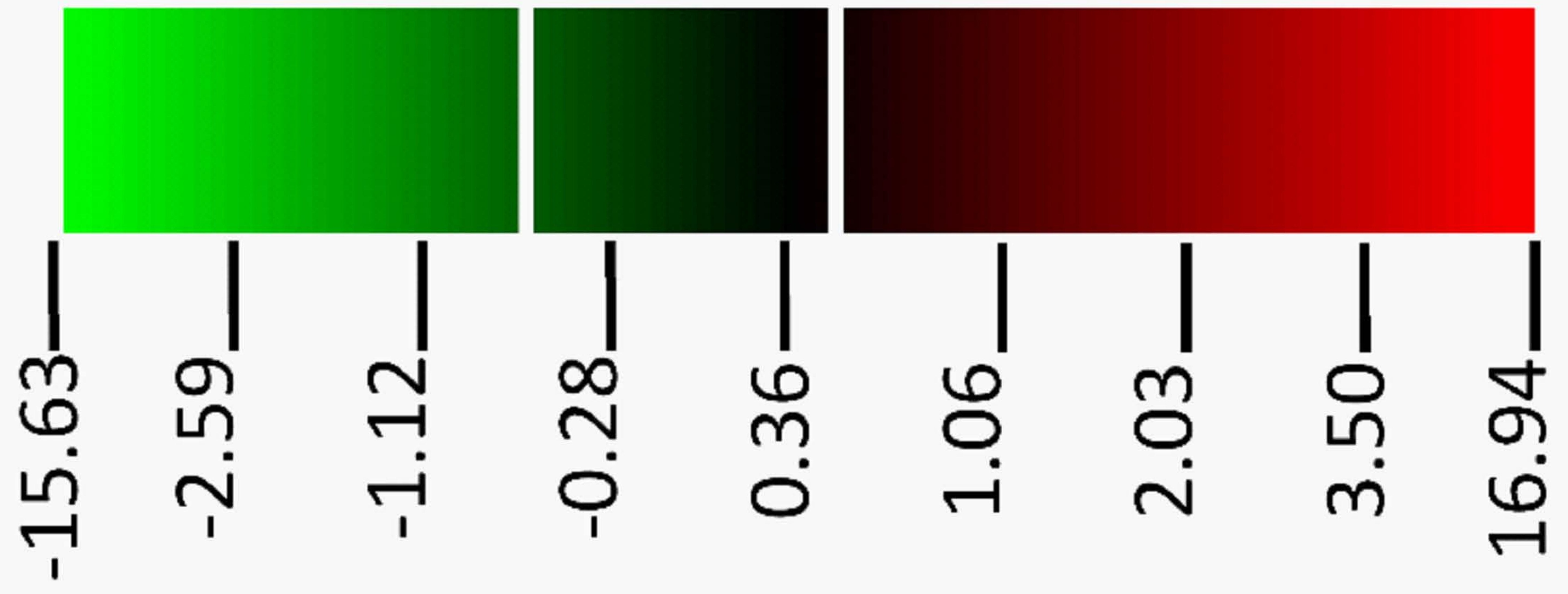

